\title{
EFFECTS OF DEXMEDETOMIDINE \\ ON ACTIVATING INTESTINAL GLIAL CELLS
}

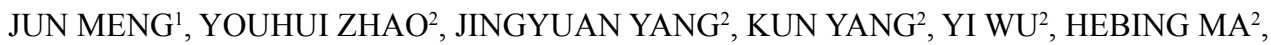 \\ and $\mathrm{KUN} \mathrm{YANG}^{2 *}$
}

${ }^{1}$ Department of Cardiovascular Surgery,

${ }^{2}$ Department of Anesthesiology,

First Affiliated Hospital of Kunming Medical University, Kunming 650032, China

\begin{abstract}
In this study, glial cell-derived neurotrophic factor GDNF was used as an evaluation index to explore the effect of dexmedetomidine, a commonly used clinical anesthetic and sedative, on the activation of intestinal glial cells. Rat intestinal glial cells were selected for in vitro experiments, cells were treated with dexmedetomidine at various concentrations for $24 \mathrm{~h}$. The expression of the glial fibrillary acidic protein (GFAP) was determined by immunofluorescence assay. The mRNA and protein levels of GDNF were measured by RT-qPCR and Western Blot, respectively. Immunofluorescence of GFAP results indicated that intestinal glial cells had slender synapses distributed on the edge of the cell body. No differences in GAFP expression were observed between dexmedetomidine treated and untreated cells. However, dexmedetomidine treatment increased the GDNF expression significantly at both mRNA and protein levels in intestinal glial cells. Dexmedetomidine can activate intestinal glial cells and increase the secretion of glial cell-derived neurotrophic factor (GDNF).
\end{abstract}

Keywords: intestinal glial cells, glial cell-derived neurotrophic factor, dexmedetomidine, intestinal mucosal barrier function

The intestine has the second-largest nervous system in the human body-Enteric nerve system (ENS). The enteric nervous system is distributed throughout the digestive tract and is composed of enteric neuron cells and enteric glial cells (EGC). The number of intestinal glial cells is 4 to 10 times that of intestinal neuronal cells $(1,2)$. In recent years, more and more evidence has shown that intestinal glial cells not only support enteric neuronal cells but also play a key role in the proliferation and repair of the intestinal epithelium and the maintenance of intestinal mucosal barrier structure and function.

Dexmedetomidine (Dex) is a new type of highly selective $\alpha-2$ adrenergic receptor agonist with central anti-sympathetic properties, which can play a role in sedation, analgesia, and anti-anxiety. Dexmedetomidine has been applied in clinical anesthesia and is widely used in intensive care and postoperative sedation.

Glial-derived neurotrophic factor (GDNF) is a signal molecule secreted by intestinal glial cells. In this study, rat intestinal glial cells were used for in vitro experiments, and GDNF was used as an evaluation index to study the role of dexmedetomidine in activating intestinal glial cells at the molecular and cellular levels.

\section{METHOD}

\section{Cells and reagents}

Rat primary intestinal glial cells were obtained from Cybertron Biotechnology (Shanghai, China). TRIzol reagent, cDNA first-strand synthesis kit, SYBR Select Master Mix antibodies specific for GDNF and GFAP were purchased from ThermoFisher (USA). Anti-GAPDH, Tubulin antibodies, and anti-mouse IgG HRP conjugated secondary antibodies were purchased from Cell signaling Technology (CST, USA).

\section{Cytotoxicity assay}

$1 \times 10^{4}$ cells were cultured in 96-well plates and incubated at $37^{\circ} \mathrm{C}$ for $24 \mathrm{~h}$. Dexmedetomidine with various concentrations $(0-200 \mu \mathrm{M})$ was added into cells for $24 \mathrm{~h}$. The cytotoxicity of dexmedetomidine was evaluated by the cell counting kit-8 (CCK8) (Donjindo) following the manufacturer's instructions. The optical density (OD) value of the wells at

* Corresponding author: e-mail: kangrong448747@163.com 
a wavelength of $450 \mathrm{~nm}$ was measured by a microplate reader (Bio-Rad). Relative cell viability rate was determined as described previously (3).

\section{Dexmedetomidine treatment}

The rat primary intestinal glial cells were seeded into 24-well plates. When cells grew to $70-80 \%$ confluence, dexmedetomidine at various concentrations was added into cells and incubated for $24 \mathrm{~h}$. The untreated cells were set as a negative control. All experiments were performed in triplicates.

\section{Immunofluorescence assays}

At $24 \mathrm{~h}$ post dexmedetomidine treatment, cells were fixed with $4 \%$ paraformaldehyde in PBS for $15 \mathrm{~min}$ at room temperature. Then cells were treated with PBS containing $0.1 \%$ Triton X-100 for 10 mins followed by blocking with PBS containing 5\% FBS for $1 \mathrm{~h}$. Subsequently, cells were incubated with a monoclonal antibody against GDNF (1: 100, ThermoFisher) for $1 \mathrm{~h}$ followed by incubated FITC-conjugated goat anti-mouse IgG (CST). Nuclear staining with DAPI was performed following manufacturer instructions (ThermoFisher). The fluorescence was observed under a Leica DMI4000 B microscope (Leica, Germany).

\section{Real-Time PCR}

Total RNA was isolated by TRIzol reagent (Life Technologies) and cDNA was synthesized by the Thermo Scientific Revert Aid First Strand cDNA Synthesis Kit (Thermo Scientific) according to the manufacturer's instruction. Quantitative PCR was performed in triplicates with SYBR Select Master Mix (Applied Biosystems) on the 7500 Real-Time PCR system (Applied Biosystems). The reaction was performed for $94^{\circ} \mathrm{C}$ for $5 \mathrm{~min}$; 40 cycles of $94^{\circ} \mathrm{C}$ for $15 \mathrm{~s}, 60^{\circ} \mathrm{C}$ for $15 \mathrm{~s}$. The gene expression relative to GAPDH was determined by the $2^{-\triangle \Delta C T}$ method. Primers were summarized in Table 1.

\section{Western blot}

The intracellular proteins were harvested by RIPA buffer (Life Technologies) and subjected to $12 \%$ sodium dodecyl sulfate-polyacrylamide gel

Table 1. Primers used in this study.

\begin{tabular}{|c|c|}
\hline Primer name & Sequence (5'-3') \\
\hline GDNF-F & GCGCTGACCAGTGACTCCAAT \\
\hline GDNF-R & GTGCCGCCGCTTGTTT ATC \\
\hline GAPDH-F & AGTGCCAGCCTCGTCTCATA \\
\hline GAPDH-R & ATGAAGGGGTCGTTGATGGC \\
\hline
\end{tabular}

electrophoresis (SDS-PAGE) and transferred onto a nitrocellulose membrane (Millipore). The membrane was blocked with 5\% nonfat dry milk in TBST, then incubated with the monoclonal antibody against GDNF (1: 3000, ThermoFisher), Tubulin (1: 3000, CST) or GAPDH (1: 3000, CST) overnight at $4{ }^{\circ} \mathrm{C}$. Subsequently, the membrane was incubated with the anti-mouse IgG HRP conjugated secondary antibody (1: 5000, CST) for $1 \mathrm{~h}$ at room temperature. The bands were developed by BeyoEcl Star Kit (Beyotime, China) and the relative expression of GDNP was expressed as GDNF/GAPDH ratio analyzed by Image J software.

\section{Elisa}

To detect secreted GDNF in the medium, the culture supernatants of cells after $24 \mathrm{~h}$ dexmedetomidine treatment were collected. The GDNF content was measured by the GDNF ELISA kit (Abcam, USA) according to the manufacturer's instructions.

\section{Statistical analysis}

Data were analyzed using statistical software (SPSS 20; IBM SPSS, USA). Data were presented as mean $\pm \mathrm{SD}$. Comparison within the group was analyzed by paired sample t-test. A P value $<0.05$ was considered statistically significant.

\section{RESULTS}

\section{Cellular toxicity of dexmedetomidine}

To exclude the possibility that cytotoxicity of dexmedetomidine has an impact on cell viability, cytotoxic effects of dexmedetomidine were evaluated by the CCK- 8 assay. As shown in Figure 1, dexmedetomidine did not show significant toxicity on rat primary intestinal glial cells up to $100 \mu \mathrm{M}$,

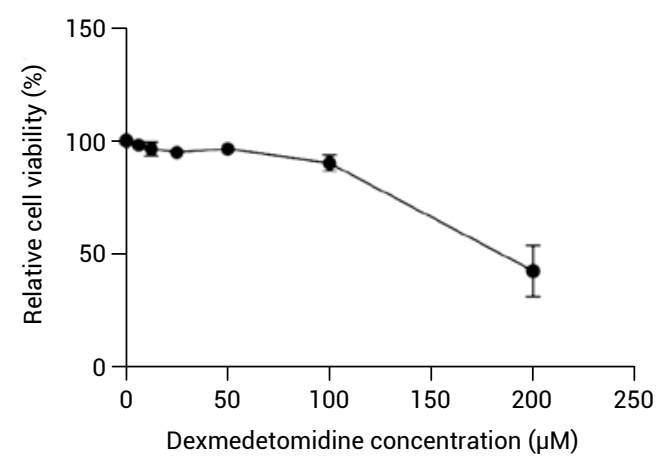

Figure 1. Cytotoxic effect of dexmedetomidine on rat primary intestinal glial cells. To determine the cytotoxicity, cells were treated with various concentrations of dexmedetomidine $(0-200 \mu \mathrm{M})$ for $24 \mathrm{~h}$. Relative cell viability was determined by CCK 8 assay and normalized to the value of $0 \mu \mathrm{M}$ group (set up as $100 \%$ ). Data are mean \pm SD from three independent experiments. 


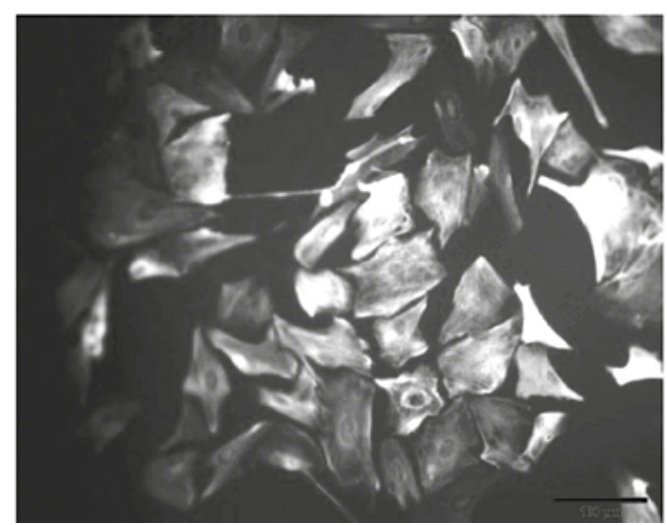

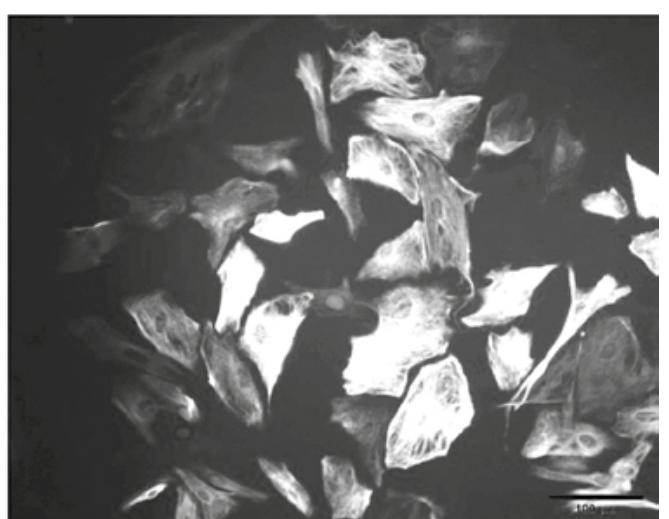

Figure 2. The effect of dexmedetomidine treatment on GFAP expression. (A) untreated group. (B) cells were treated with $100 \mu \mathrm{M}$ of dexmedetomidine.

the average cell survival rate was above $90 \%$, and no difference in cell morphology compared with mock-treated cells was observed (data not shown). Therefore, the highest concentration of $100 \mu \mathrm{M}$ was selected for further experiments.

\section{The effect of dexmedetomidine treatment on GFAP expression}

Since intermediate filament proteins-intestinal glial fibrillary acidic protein (GFAP) and calciumbinding protein $\mathrm{S} 100$ protein are expressed in intestinal glial cells, the morphology of intestinal glial cells was evaluated by GFAP expression. As shown in Figure 2, no significant differences in GFAP expression were observed between dexmedetomidinetreated cells and control cells. Intestinal glial cells presented clear, continuous, and elongated synapses

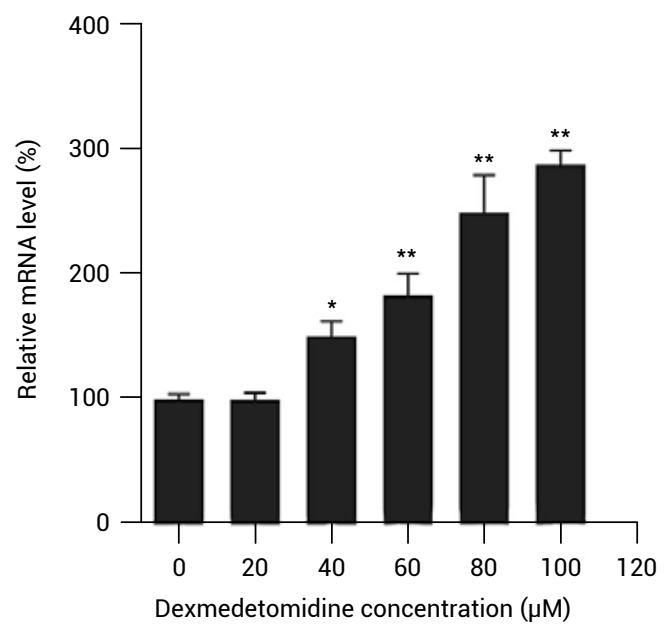

Figure 3. Effect of dexmedetomidine on mRNA expression of GDNF. Data were normalized to values of $0 \mathrm{mM}$ group (set up as $100 \%$ ). Error bars indicate SD from three independent experiments and statistically significant differences are indicated by asterisks $(* \mathrm{P}<0.05, * * \mathrm{P}<0.01)$. around the nucleus, distributed at the edge of the cell body, similar in shape to astrocytes in the central nervous system.

\section{GDNF mRNA levels after dexmedetomidine treatment}

To evaluate the effect of dexmedetomidine on GDNF mRNA level, intestinal glial cells were treated with different concentrations of dexmedetomidine for $24 \mathrm{~h}$, GDNF mRNA was measured by RT-PCR. As shown in Figure 3, dexmedetomidine treatment increased the GDNF mRNA level in a dose-dependent manner, indicating that dexmedetomidine treatment significantly upregulated GDNF mRNA expression compared to the control group $(\mathrm{P}<0.05)$.

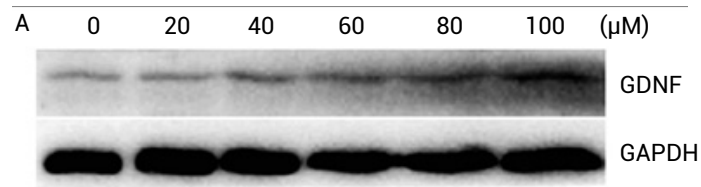

B

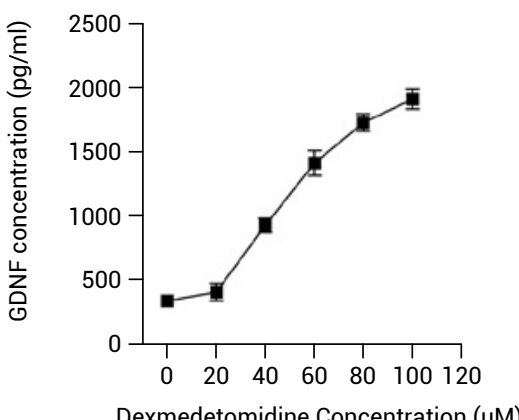

Figure 3A. Effect of dexmedetomidine on GDNF protein expression. (A) The intracellular GDNF was detected by western blot analysis. (B) The secreted GDNF was measured by the GDNF Elisa kit. 


\section{Dexmedetomidine enhanced GDNF protein expression}

To further characterize dexmedetomidine function on GDNF expression, the intracellular and extracellular expression of GDNF were determined. As shown in Figure 3A, the protein level of GDNF after dexmedetomidine treatment was significantly higher than in the untreated group. The ratio of GDNF to GAPDH was $0.40 \pm 0.07$ and $0.75 \pm 0.12$ in dexmedetomidine treated $(100 \mu \mathrm{M})$ and untreated groups, respectively. In addition, the secreted GDNF was measured by an ELISA kit, the results were in agreement with the observation of intracellular expression. Our results indicated that dexmedetomidine treatment significantly induced both intracellular and extracellular GDNF protein expression.

\section{DISCUSSION}

Traumas such as hemorrhage or hemorrhagic shock during surgery cause damage to the intestinal mucosal barrier function (4). The intestinal glial cells play an important regulatory role in the repair of the intestinal mucosal barrier. Intestinal glial cells provide nutrition and support to intestinal epithelial cells. Intestinal glial cells also cause phenotypic changes in the intestinal mucosal barrier by regulating the expression and secretion of neural signal molecules and maintain the integrity of the intestinal mucosal barrier (5).

Intestinal glial cells derived from the neuroectoderm are important components of the enteric nervous system. Intestinal glial cells are mainly distributed in the submucosal plexus of the intestinal wall and the intestinal myenteric plexus (2). Its morphology and function are the same as those of the central nervous system. Similar to astrocytes, glial fibrillary acidic protein (GFAP) and calciumbinding protein S100 are the most commonly used markers of intestinal glial EGC (2). Therefore, we used GFAP as a marker to observe the morphology of intestinal glial cells.

The terminal feet of intestinal glial cells EGC under the mucosa of the intestinal wall are in close contact with intestinal epithelial cells. EGC cells secrete neural signal molecules and regulate the intestinal barrier function through these neural signal molecules. The signal molecules secreted by intestinal glial cells include glial cell-derived neurotrophic factor (GDNF), GSNO (glial-derived snitrosoglutathione) (2, 6-9). In the current study, GDNF was selected as the detection index to evaluate the function of dexmedetomidine on activating intestinal glial cells.
Dexmedetomidine is a highly selective $\alpha-2$ adrenergic receptor agonist. Dexmedetomidine inhibits the release of norepinephrine after activating the receptor and has central anti-sympathetic properties, meanwhile, it exerts sedative, analgesic, and antianxiety effects, and does not significantly inhibit breathing (10). Therefore, dexmedetomidine is increasingly used in clinical anesthesia, intensive care, and postoperative sedation. Clinically, the level of serum dexmedetomidine concentration is $\mu \mathrm{mol} / \mathrm{L}$ when applied in clinical anesthesia and sedation of critically ill patients. Refer to the dose screening of dexmedetomidine in vitro (11), we further determined the toxicity of dexmedetomidine on rat primary intestinal glial cells, since the cell viability was above $90 \%$ when treated with $100 \mu \mathrm{M}$ of dexmedetomidine. The highest concentration of dexmedetomidine was set as $100 \mu \mathrm{M}$ in this study.

Our results demonstrated that similar to the morphology of astrocytes in the central nervous system, intestinal glial cells presented long and thin synapses distributed on the edge of the cell body. The dexmedetomidine treatment showed no significant effect on the expression of the intestinal glial fibrillary acidic protein (GFAP). 24 hours post-treatment, the GDNF expression level of intestinal glial cells EGC significantly increased at both mRNA and protein levels, indicating that dexmedetomidine activated intestinal glial cells and the secreted signal molecule GDNF increased dramatically. The previous study demonstrated that intraperitoneal injection of GDNF in genetically mutant rats with intestinal glial cell defects up-regulated the expression of intestinal epithelial cell tight junction proteins ZO-1 and Occludin (6), and significantly reduced the permeability of the rat intestinal mucosal barrier and restored the intestinal mucosal barrier function. In addition, GDNF showed a strong antiapoptotic effect on intestinal epithelial cells (12). Our results showed that dexmedetomidine activated intestinal glial cells and induced secretion of GDNF. Dexmedetomidine is a commonly used clinical anesthetic and sedative with a protective effect on the intestinal mucosal barrier (13-15). Our in vitro experiments demonstrated that the protective effect of dexmedetomidine on intestinal mucosal barrier damage may be related to the activation of intestinal glial cells to promote GDNF expression. The mechanism of dexmedetomidine activating intestinal glial cells remains unclear. Previous studies have demonstrated that dexmedetomidine promotes the expression of $\alpha 7$-nicotinic acetylcholine receptor ( $\alpha 7-n A C h R)$ in the hippocampus of rats (16). Since $\alpha 7-n A C h R$ also expresses on intestinal glial cells, we speculate that 
dexmedetomidine may activate intestinal glial cells through a cholinergic pathway mediated by this receptor. The specific mechanism needs further confirmation in the future.

\section{Acknowledgments}

This work was supported by the National Natural Science Foundation of China (Grant no. 81760348) and the Yunnan health training project of highlevel talents (Grant no. D-2018019).

\section{Conflict of interest}

The authors declare no conflicts of interest.

\section{REFERENCE}

1. Ochoa-Cortes F., Turco F., Linan-Rico A., Soghomonyan S., Whitaker E., et al.: Inflamm. Bowel Dis. 22, 433 (2016).

2. Neunlist M., Rolli-Derkinderen M., Latorre R., Van Landeghem L., Coron E., et al.: Gastroenterology 147, 1230 (2014).

3. Cui J., Hie J., Gao M., Zhou H., Chen Y., et al.: Antivir. Ther. 20, 565 (2015).

4. Meng J., Huang Y.C., Huang J., Yang K.: J. Trauma Acute Care Surg. 80, 972 (2016).

5. Aube A.C., Cabarrocas J., Bauer J., Philippe D., Aubert P., et al.: Gut 55, 630 (2006).
6. Savidge T.C., Newman P., Pothoulakis C., Ruhl A, Neunlist M., et al.: Gastroenterology 132, 1344 (2007).

7. Flamant M., Aubert P., Rolli-Derkinderen M., Bourreille A., Neunlist M.R., et al.: Gut 60, 473 (2011).

8. Cheadle G.A., Costantini T.W., Lopez N., Bansal V., Eliceiri B.P., et al.: PLoS One 8, e69042 (2013).

9. Bauman B.D., Meng J., Zhang L., Louiselle A., Zheng E., et al.: J. Surg. Res. 219, 214 (2017).

10. Weerink M.A.S., Struys M.M.R.F., Hannivoort L.N., Barends C.R.M., Absalom A.R., et al.: Clin. Pharmacokinet. 56, 893 (2017).

11. Gao J.D., Sun Z.H., Xiao Z.Y., Du Q., Niu X., et al.: Br. J. Anaesth. 123, 827 (2019).

12. Zhang D.K., He F.Q., Li T.K., Pang X.H., Cui D.J., et al.: J. Pathol. 222, 213 (2010).

13. Zhang X.Y., Liu Z.M., Wen S.H., Li Y.S., Li Y., et al.: Anesthesiology 116, 1035 (2012).

14. Yeh Y.C., Wu C.Y, Cheng Y.J., Liu C.M., Hsiao J.K., et al.: Anesthesiology 125, 355 (2016).

15. Wang Z.X., Huang C.Y., Hua Y.P., Huang W.Q., Deng L.H., et al.: Br. J. Anaesth. 112, 1055 (2014).

16. Xu K.L., Liu X.Q., Yao Y.L., Ye M.R., Han Y.G., et al.: Biochem. Biophys. Res. Commun. 495, 421 (2018). 